\title{
Society of Hair Testing guidelines for drug testing in hair $\mathrm{r}^{\text {i⿱ }}$
}

\author{
Gail A.A. Cooper ${ }^{\mathrm{a}, *}$, Robert Kronstrand ${ }^{\text {b,c }}$, Pascal Kintz ${ }^{\mathrm{d}}$ \\ ${ }^{a}$ Forensic Medicine and Science, University of Glasgow, G12 8QQ Glasgow Scotland \\ ${ }^{\mathrm{b}}$ Department of Forensic Genetics and Forensic Toxicology, National Board of Forensic Medicine, SE-58758 Linköping, Sweden \\ ${ }^{c}$ Department of Medical and Health Sciences, Faculty of Health Sciences, Linköping University, SE-58185 Linköping, Sweden \\ ${ }^{\mathrm{d}}$ X-Pertise Consulting, 84 route de Saverne, 67205 Oberhausbergen, France
}

\section{A R T I C L E I N F O}

\section{Article history:}

Received 24 January 2011

Accepted 24 January 2011

Available online $\mathrm{xxx}$

\section{Keywords:}

Hair testing

Guidelines

\begin{abstract}
A B S T R A C T
The Society of Hair Testing (SoHT) Guidelines for Drug Testing in Hair provide laboratories with recommended best practice guidelines whether they are currently offering drug testing in hair, or plan to offer a hair testing service in the future. The guidelines include reference to recommended sample collection and storage procedures, through sample preparation, pre-treatment and analysis and the use of cut-offs.
\end{abstract}

Crown Copyright @ 2011 Published by Elsevier Ireland Ltd. All rights reserved.

\section{Introduction}

Founded in 1995, the Society of Hair Testing (SoHT) [1] has a worldwide network of members involved in hair testing and has published several guidance documents relating to the examination of drugs and doping agents in hair [2-5]. Hair testing is well established as a complementary technique with a range of applications in both clinical and forensic toxicology. The advantage of hair is its ability to provide a historical profile of an individuals' exposure to drugs following chronic use or a single exposure.

The SoHT understands the importance of setting industry standards to ensure the future of hair testing and its continued acceptance as evidence in court and actively encourages its members to participate in the Society's annual proficiency testing scheme.

The SoHT recognises the international standards ISO/IEC 17025:2005 [6] and ILAC-G19 [7] and acknowledges the European guidelines for hair in relation to workplace drug testing (http:// www.ewdts.org) [8], the hair guidelines published by the Society of Toxicological and Forensic Chemistry (http://www.gtfch.org) [9] and other national drug testing guidelines for forensic toxicologists.

These guidelines for drug testing in hair were reviewed and approved by the Board of the Society of Hair Testing at the annual Board meeting held in Sevilla in January 2011.

\footnotetext{
This paper is part of the special issue entitled: Selected papers from the Chamonix 2011 Society of Hair Testing Meeting, Guest-edited by Pascal Kintz.

* Corresponding author. Tel.: +44 141330 4574; fax: +44 1413304602.

E-mail address: gail.cooper@glasgow.ac.uk (Gail A.A. Cooper).
}

\section{Scope}

The purpose of this document is to provide hair testing laboratories and national accreditation bodies with internationally accepted best practice guidelines for drug testing in hair. In addition, the guidelines aim to provide a useful guide to laboratories planning to or currently offering hair testing services so that they may fully understand the requirements involved with establishing a quality-enhanced hair testing service.

\section{Applications of hair testing}

The role of hair testing as an alternative or complementary matrix has expanded across the spectrum of toxicological investigations and as a consequence samples of hair are routinely collected during criminal investigations (drug-related deaths, drug-facilitated crime, child protection, etc.) and for monitoring drug misuse such as drug rehabilitation programmes, workplace drug testing and driving licence re-granting.

Hair is a strong, stable tissue less affected by adulterants or short-term abstinence and has the advantage over traditional matrices (e.g. blood or urine) of being able to confirm long-term exposure to drugs over a period of weeks to months depending on the length of hair collected.

Routes of incorporation from blood capillaries at the root bulb, sweat, sebum and external contamination may all contribute to the drug concentrations measured in hair and therefore must be considered when interpreting the findings.

\subsection{Drug-related deaths}

Drug levels detected in post-mortem blood provide information regarding the short-term drug use of the decedent prior to death, 
while hair analysis provides an invaluable source of information relating to antecedent drug use history in the months prior to death. Establishing evidence of chronic drug use through hair analysis can greatly aid the investigative process and help establish the role of drugs with respect to the cause of death. Establishing abstinence in the weeks to months prior to death provides supporting evidence of a lack of tolerance to the drugs implicated in the cause of death. The collection and analysis of hair roots has the potential to provide information relating to acute poisoning. The analysis of hair roots may provide crucial information in cases of advanced decomposition or delayed death where there is limited availability of samples suitable for analysis and justifies the additional effort required to prepare hair roots.

\subsection{Drug facilitated crime (DFC)}

Drugs most commonly associated with drug facilitated crimes typically cause sedation and amnesia, e.g. benzodiazepines. Victims may not report the crime for a considerable time after the event, compromising the ability of blood or urine samples to identify the drug administered. Hair provides an opportunity to identify the drug or drugs many months later.

This presents investigators with a considerable challenge as the identification of a single dose requires sensitive instrumentation to detect low pg/mg levels in hair. Gamma hydroxybutyrate (GHB) requires special mention as it is an endogenous compound present in low concentrations in the body but is also widely available and associated with the club scene and in cases of drug facilitated sexual assault (DFSA). As GHB is endogenous, GHB may be detected in hair at low concentration and as such the presence of GHB alone is insufficient to confirm exogenous administration. An elevation of the GHB concentration in the hair segment consistent with the approximate timeframe of the incident is required to differentiate between endogenous and exogenous GHB.

\subsection{Child custody}

Hair analysis provides a means of evaluating long-term drug exposure in infants and children and potential sources of the exposure that must be considered. Mothers who continue to misuse drugs and alcohol during pregnancy expose the unborn foetus as demonstrated by drug positive meconium and hair collected from the newborn. Drugs are also transfered in maternal milk to babies who are breastfed, and young children who are crawling or starting to walk can access drugs or drug residue on contaminated surfaces. Additional potential sources include smoke in the atmosphere and sweat from close contact with adults or siblings who use drugs.

When considering the potential sources of a positive hair sample from an infant or child, the contribution from gestational exposure must be explored. Many infants are born with hair of varying lengths and although many lose their hair in the months after birth, some will not lose their hair. If the child has not had their hair cut since birth and the mother was known to use drugs during her pregnancy, any drugs identified in the distal region of the hair must be attributed to exposure in the womb. Full details relating to the infant or child's hair and age should be requested and interpreted with caution. Average hair growth rates which apply to adults should not be assumed for infants and children whose growth rates may vary considerably.

\subsection{Chronic excessive alcohol consumption [5]}

The use of specific diagnostic biomarkers for chronic excessive alcohol consumption, ethyl glucuronide (EtG) and fatty acid ethyl esters (FAEEs), have been investigated in various matrices including hair. The identification of either EtG or FAEEs in hair, at concentrations above established cut-offs, is acceptable for assessing chronic alcohol consumption, however, the identification of both markers is encouraged. EtG and FAEEs are affected by cosmetic treatments, however, their incorporation into hair is not affected by hair pigmentation. The role of contamination from cosmetics should be considered when interpreting the presence of alcohol biomarkers in hair $[10,11]$.

\section{Sample collection}

Collecting head hair is less intrusive and causes less embarrassment than observed urine collection and hair does not require refrigeration and can be stored indefinitely. Drugs are relatively stable in hair and as such a second representative hair sample can be collected and analysed.

It is accepted that head hair grows at an average rate of $1 \mathrm{~cm}$ each month [12] and a sample cut from the posterior vertex region of the head, close to the scalp is preferred as this region of the scalp is associated with least variation in growth rates. The amount of hair required for analysis is a "lock of hair" or a pencil thickness of hair. It is important to collect sufficient hair in order to carry out routine tests and to allow for a repeat analysis or confirmation test by a second laboratory.

Concerns are often raised in relation to leaving a visible "bald patch" of particular concern with small children or individuals with baldness or thinning hair. In these cases, collection of several smaller hair samples from multiple sites, focusing where possible around the posterior vertex region is acceptable.

Head hair is the preferred sample, however, if head hair is not available alternative collection sites should be considered including pubic, underarm and beard hair. Collection of intimate samples requires consideration for the privacy of the donor while ensuring that the integrity of the collection process is not compromised. Growth rates and dormancy characteristics of hair from these alternate sites, differs from head hair.

Cosmetic treatments whether regular shampooing, dyeing or relaxing/bleaching, may have a deleterious effect on the drug concentrations in hair but to different extents. Aggressive bleaching treatments reduce drug concentrations and therefore all declared hair treatments must be considered when reviewing the findings of a hair test.

\subsubsection{Recommendations for sample collection}

- The collection of hair samples should be undertaken by a competent individual within a secure contamination-free facility with access restrictions in place.

- A hair collection kit with clear instructions for collection should be provided and the collector must observe chain of custody procedures and wear gloves when handling hair.

- The collection kit should include:

- Chain of custody form

- Foil and collection envelope

- Security seal

- Evidence bag

- Transportation envelope

- Instructions for the collection of a hair sample

- The colour, length, site of collection and any obvious cosmetic treatments should be recorded.

- The head hair sample should be aligned with the root end of the sample clearly identified and secured, e.g. with foil as illustrated in Fig. 1.

- Hair samples must be stored in a dry, dark environment at room temperature, away from direct sunlight. Hair samples should not 


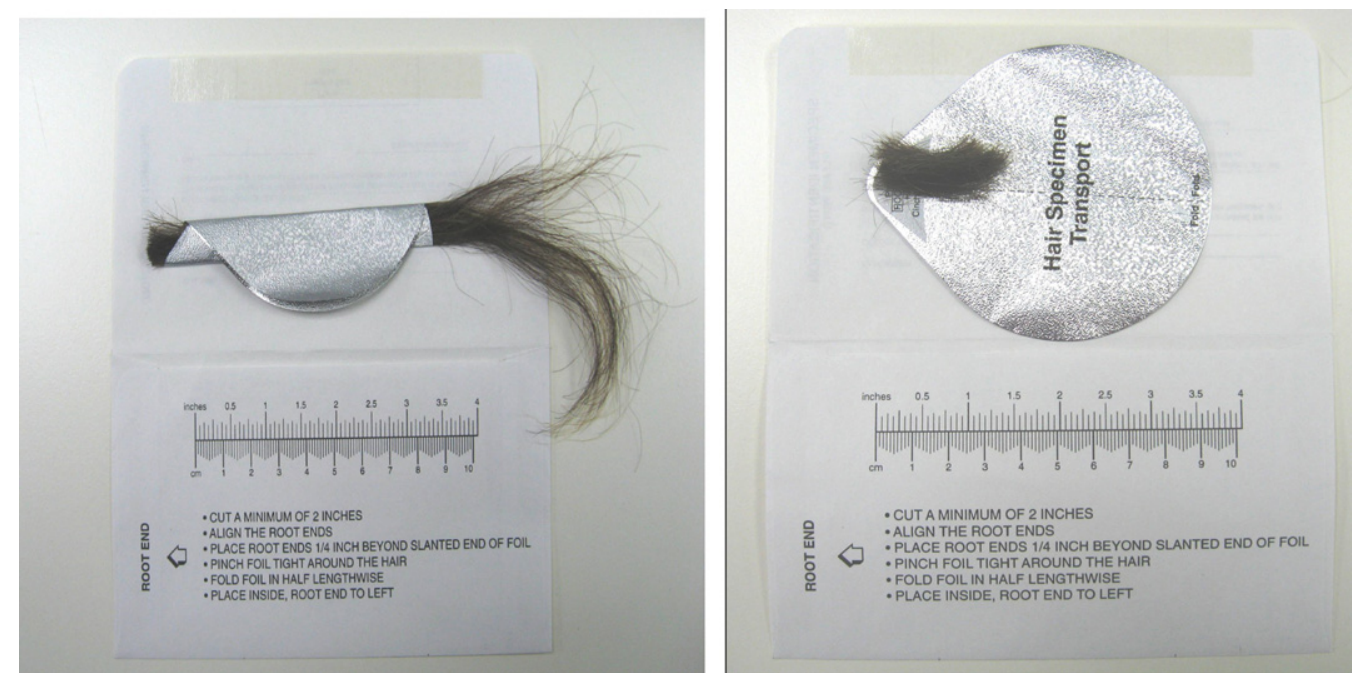

Fig. 1. Cut hair (long and short) aligned with the root end identified and then secured with foil. Picture courtesy of Forensic Medicine and Science, University of Glasgow, Copyright 2011.

be stored in the refrigerator or freezer, since swelling may occur and drug may be lost.

- Hair samples that are wet on submission MUST be dried before storage and analysis.

\subsection{Collection of post-mortem hair samples}

The analysis of post-mortem hair samples can provide important information relating to the drug use history of the deceased. The collection procedures detailed above apply in post-mortem cases, however, a standard hair collection kit foil and envelope may be inappropriate depending on the condition of the deceased's hair and an alternative more robust collection vessel required. The analysis of hair roots may help provide additional information relating to the deceased's drug use prior to death.

\subsubsection{Recommendations for collection of post-mortem hair samples}

- Hair should be collected at the beginning of the autopsy.

- If information is required relating to recent drug use, the collection of a pulled hair sample with roots is recommended in addition to the collection of a cut hair sample.

\subsection{Collection of hair samples in drug-facilitated crime (DFC)}

The collection of hair samples in cases of alleged DFC is time critical as the complainant may not report the incident for several days or a number of weeks or months in some instances. Details relating to the time elapsed between the incident and the date of report are essential when deciding on the appropriate date(s) of sample collection. Chemical treatment of hair after the incident will adversely affect the suitability of hair as a testing matrix.

\subsubsection{Recommendations for collection of hair samples in DFC}

- A hair sample should be collected a minimum of 4-6 weeks after the alleged incident.

- If the sample tests positive, it is recommended that a second hair sample is collected to corroborate the findings.

- The complainant must not have any cosmetic treatments or cut their hair between hair collections or until the investigations have concluded.

\section{Sample preparation}

The preparation of hair samples involves a number of steps including washing, segmentation (optional) and obtaining a representative sample from the material available.

\subsection{Segmental analysis}

Depending on the type of case, different strategies for segmentation may apply. Head hair cut into measured segments of between 10 and $30 \mathrm{~mm}$ can provide a more detailed historical profile of an individual's drug exposure. In cases of alleged DFC, including drug-facilitated sexual assault (DFSA), where a single administration of GHB or another central nervous system depressant drug is suspected, the analysis of even smaller segments may help improve the establishment of a positive hair test in a narrower timeframe. The accuracy of segmental analysis depends on both the sampling and the segmentation procedure at the laboratory. In addition, drug concentrations in hair may decrease from the root to distal end of the hair, through natural "wash-out" of drugs from hair.

\subsubsection{Recommendations for segmental analysis}

- Only hair cut from the scalp and aligned with the root end identified should be subjected to segmental analysis.

\subsection{Washing}

Washing of hair samples prior to analysis has two main purposes. First, to remove hair care products, sweat, sebum or surface material (e.g. skin cells, head lice, body fluids, etc.) that may interfere with the analysis or that may reduce extraction recovery. Second, to remove potential external contamination of drugs from the environment.

Standardised wash procedures that will effectively remove any trace of external contamination without actively removing the drugs incorporated into the hair are not currently available and as such the possible role of external contamination must be considered when interpreting hair testing findings. However, several approaches have been described to discriminate between external contamination and drugs incorporated through ingestion. It is generally accepted that organic solvent such as methylene chloride or acetone will remove only surface contamination 
whereas aqueous solutions or methanol will swell the hair and extract drugs from within the hair matrix.

\subsubsection{Recommendations for washing}

- The laboratory should have a procedure for washing hair samples prior to analysis.

- The procedure should include washing steps with both organic solvent and aqueous solutions.

- The laboratory should investigate to what extent their wash procedure removes surface contamination.

- Hair samples heavily soiled with body fluids may require additional clean-up procedures.

\subsection{Sample homogeneity}

Hair as a matrix differs from body fluids such as blood or urine as both are easily aliquoted for analysis. Whether a sample of hair has been cut into smaller segments prior to analysis or analysed as one bulk sample, it is important that the sample is homogeneous and the portion taken is a representative sample.

\subsubsection{Recommendations for sample homogeneity}

- Hair samples that have been washed and dried should be cut into smaller pieces or milled to a powder, and then typically 10$50 \mathrm{mg}$ of hair accurately weighed prior to analysis.

\section{Testing procedures}

Hair analysis involves an initial pre-treatment step to release the drugs from within the hair matrix. The resulting extract can be analysed directly using screening techniques or will require further clean-up using either liquid-liquid extraction (LLE) or solid-phase extraction (SPE) prior to confirmation procedures.

\subsection{Incubation and extraction}

Method efficiency is compromised significantly through use of unsuitable extraction procedures not targeted to specific drugs. It is important to consider the potential deleterious effect on the drugs in question when choosing the extraction solutions. In vitro hydrolysis of labile compounds such as cocaine and heroin has been reported under acidic or alkaline conditions. Incubation procedures with methanol on the other hand do not have the same issues with hydrolysis but recoveries are lower.

\subsubsection{Recommendations for incubation and extraction}

- Laboratories introducing new hair testing methodologies must investigate the efficiency of different incubation conditions to optimise their in-house extraction procedures, while minimising hydrolysis of labile drugs.

\subsection{Screening techniques}

Screening for a range of drugs in hair is achieved through immunological or chromatographic methodologies. Immunoassays commonly used for rapid screening of drugs in biological fluids are also available for hair. The use of chromatographic techniques with tandem mass spectrometry and time of flight (TOF) capabilities, although less practical for screening large numbers of samples, have the advantage of being able to simultaneously screen and identify a wider range of analytes in a single analysis. This is a significant advantage when dealing with small sample volumes.

\subsubsection{Recommendations for screening techniques}

- Laboratories must ensure that screening assays have sufficient sensitivity to detect low drug levels in hair and are targeted to reflect the drug profile in hair.

- The hair matrix should not interfere with the immunoassay.

- All presumptive positive immunoassay screening tests must be confirmed using a more specific test for the target analyte, e.g. mass spectrometry.

\subsection{Confirmation techniques}

Separation and detection of the analytes of interest is achieved using chromatographic methods hyphenated with mass spectrometry, e.g. gas chromatography-mass spectrometry (GC-MS) and liquid chromatography-mass spectrometry (LC-MS). Drug levels in hair are considerably lower than those found in routinely tested matrices such as blood and urine and as such single and tandem mass spectrometry are employed for confirmation of drugs in hair.

\subsubsection{Recommendations for confirmation techniques}

- Laboratories must ensure that confirmation techniques have sufficient sensitivity for low drug levels found in hair and are targeted to reflect the drug profile in hair.

- Confirmation methods should include the determination of the parent drug and metabolite(s), where applicable, to help distinguish between contamination and ingestion.

\subsection{Method sensitivity and cut-offs}

The sensitivity of a method will decide whether it is appropriate for a certain type of case and whether it is suitable to provide an answer to the case-specific question. Based on previous guidelines and the available literature on drug concentrations in hair from drug users and patients we recommend cut-offs to enable identification of chronic drug use (Table 1). Cut-offs to identify chronic excessive alcohol consumption are available in the SoHT consensus document [5].

When hair analysis is utilised in DFC cases, lower cut-offs should be employed. In general, concentrations in the low picogram per milligram range are detected after a single intake of a drug, presenting a challenge for analytical procedures as well as interpretation. It is accepted that different washing procedures

Table 1

Recommended cut-offs for substances and metabolites in hair to identify use.

\begin{tabular}{|c|c|c|c|}
\hline \multicolumn{3}{|l|}{ Screening } & \multirow{2}{*}{$\begin{array}{l}\text { Confirmation } \\
\text { Cut-off (ng/mg) }\end{array}$} \\
\hline Group & Cut-off (ng/mg) & Target analyte & \\
\hline Amphetamines & 0.2 & $\begin{array}{l}\text { Amphetamine } \\
\text { Methamphetamine } \\
\text { MDA } \\
\text { MDMA }\end{array}$ & $\begin{array}{l}0.2 \\
0.2 \\
0.2 \\
0.2\end{array}$ \\
\hline Cannabinoids & 0.1 & $\begin{array}{l}\text { THC } \\
\text { THC-COOH }\end{array}$ & $\begin{array}{l}0.05 \\
0.0002\end{array}$ \\
\hline Cocaine & 0.5 & $\begin{array}{l}\text { Cocaine } \\
\text { BZE, EME, CE, NC }\end{array}$ & $\begin{array}{l}0.5 \\
0.05\end{array}$ \\
\hline Opiates & 0.2 & $\begin{array}{l}\text { Morphine } \\
\text { Codeine } \\
\text { 6-acetylmorphine }\end{array}$ & $\begin{array}{l}0.2 \\
0.2 \\
0.2\end{array}$ \\
\hline Methadone & 0.2 & $\begin{array}{l}\text { Methadone } \\
\text { EDDP }\end{array}$ & $\begin{array}{l}0.2 \\
0.05\end{array}$ \\
\hline Buprenorphine & 0.01 & $\begin{array}{l}\text { Buprenorphine } \\
\text { Norbuprenorphine }\end{array}$ & $\begin{array}{l}0.01 \\
0.01\end{array}$ \\
\hline
\end{tabular}


will affect the remaining amount of drug in the hair and the recovery is further compromised by the extraction procedure.

\subsubsection{Recommendations for method sensitivity and LLOQ's}

- For DFC cases the laboratory should verify that their methods are sensitive enough to detect a single intake of a drug.

- For endogenous compounds (e.g. GHB, cortisol, testosterone, etc.) segmental analysis should always be carried out to enable discrimination between endogenous and exogenous levels.

\subsection{Quality assurance and quality control}

Implementation of quality assurance is recognised as a fundamental principle for all testing laboratories and accreditation to the international standard ISO/IEC 17025 an industry requirement. A number of ISO documents are available to provide guidance (http://www.iso.org) with validation requirements in addition to publications specifically targeted for forensic and clinical toxicology method validation $[13,14]$.

Standard validation parameters include linearity, limits of detection and quantitation and precision. Laboratories wishing to attain ISO/IEC 17025 accreditation must also investigate the robustness of the test method, investigate analyte stability, bias and estimate uncertainty in measurement.

Accrediting methods for the analysis of drugs in hair represents a significant challenge to the analyst with respect to the limited availability of certified reference materials available for use as external quality controls. Participation in external proficiency testing programmes is recommended for all laboratories offering hair testing services and is particularly important as a means of assessing the performance of in-house methods. There are currently three European proficiency testing schemes for hair, the Society of Hair Testing (SoHT), HAIRVEQ and the German Society of Toxicological and Forensic Chemistry (GTFCh). The benefit of these schemes is that the samples are prepared using hair from known drug users.

Ensuring the performance of analytical methods may require use of internal quality control samples. Ideally, quality control samples should be prepared from "authentic" hair samples collected from known drug users, however, the preparation of controls in-house can be problematic with issues surrounding homogeneity and lack of availability of old case samples.
A list of current suppliers of quality control material, using both authentic and fortified drug-free hair, is maintained on the Society website (http://www.soht.org).

\subsubsection{Recommendations for $Q A$ and $Q C$}

- The validation of methodologies employed for the analysis of drugs in hair should use authentic hair samples to effectively evaluate the extraction efficiency of the method.

- Laboratories offering hair testing services should enrol in proficiency testing schemes that use authentic hair samples.

- Both negative and positive hair quality controls must be analysed with each batch of case samples.

- Laboratories must provide data to support their technical competence in generating valid test results.

\section{References}

[1] Society of Hair Testing Website: http://www.soht.org.

[2] Society of Hair Testing, Statement of the society of hair testing concerning the examination of drugs in human hair, Forensic Sci. Int. 84 (1997) 3-6.

[3] Society of Hair Testing, Consensus of the society of hair testing on hair testing for doping agents, http://www.SOHT.org/html/Statements.html.

[4] Society of Hair Testing, Recommendations for hair testing in forensic cases, Forensic Sci. Int. 145 (2004) 83-84.

[5] P. Kintz, Consensus of the Society of Hair Testing on hair testing for chronic excessive alcohol consumption, Forensic Sci. Int. 196 (1-3) (2010) 2.

[6] ISO/IEC 17025:2005 general requirements for the competence of testing and calibration laboratories, Incorporating Corrigendum No.1. Published under the authority of the Standards Policy and Strategy Committee on 29 June 2005, ISBN:0 580463303.

[7] ILAC-G19: 2002 Guidelines for Forensic Science Laboratories, 2002.

[8] R. Agius, P. Kintz, Guidelines for European workplace drug and alcohol testing in hair, Drug Test. Anal. 2 (2010) 367-376.

[9] Appendix C. Requirements for the examination of hair samples. Directive GTFCh for quality assurance in forensic toxicological investigations [Version 01-published 01 June 2009].

[10] L. Morini, A. Zucchella, A. Polettini, L. Politi, A. Groppi, Effect of bleaching on ethyl glucuronide in hair: an in vitro experiment, Forensic Sci. Int. 198 (1-3) (2010) 23-27.

[11] J. Gareri, B. Appenzeller, P. Walasek, G. Koren, Impact of hair-care products on FAEE hair concentrations in substance abuse monitoring, Anal. Bioanal. Chem. 400 (1) (2011) 183-188.

[12] M.R. Harkey, Anatomy and physiology of hair, Forensic Sci. Int. 63 (1993) 9-18.

[13] F.T. Peters, H.H. Maurer, Bioanalytical method validation and its implications for forensic and clinical toxicology-a review, Accredit. Qual. Assur. 7 (2002) 441-449.

[14] F.T. Peters, O.H. Drummer, F. Musshoff, Validation of new methods, Forensic Sci. Int. 165 (2007) 216-224. 